Compte tenu du régime du budget global en vigueur en Allemagne, chaque cabinet médical se voit attribuer un budget personnel. Même le strict respect de ce budget ou le fait de n'avoir pas facturé, ou à peine, les prestations en excès ne met pas le médecin à l'abri d'un contrôle d'économicité. Et si, lors d'un tel contrôle, il lui est impossible de justifier en détail même des années plus tard les traitements des patients, le médecin s'expose à des actions récursoires, à savoir à des demandes de restitution pouvant porter sur des sommes à six chiffres [1]. L'article de Britta Beeger ci-après, paru à I'origine dans la Frankfurter Allgemeine Zeitung le 3 mai 2018, montre les difficultés auxquelles sont confrontés les médecins de campagne dans le land de Hesse.

Jürg Schlup

Dr méd., président de la FMH

\title{
Les souffrances du médecin de campagne
}

\section{Britta Beeger}

\author{
Quelques médecins du land de Hesse, en Allemagne, doivent restituer des sommes \\ astronomiques pour avoir traité trop de patients à domicile. Leur conseil aux \\ jeunes: «Ne devenez surtout pas médecin de campagne!»
}

Il y a des choses que Silvia Steinebach considère comme évidentes. Par exemple le fait de maintenir des patients atteints de maladies incurables le plus longtemps possible chez eux. «Personne n'a envie de mourir à l'hôpital», nous lance la praticienne de Hainzell, petite bourgade à proximité de Fulda. Lorsqu'un de ses patients souffre d'une insuffisance cardiaque ou rénale et peine à sortir de chez lui, elle fait le déplacement. Financièrement, ce n'est pas rentable, car elle ne facture que 38,50 euros. Elle gagnerait beaucoup plus en restant au cabinet. Mais pas question pour elle de laisser ses patients âgés livrés à eux-mêmes.

\section{Astreinte à rembourser 50000 euros pour avoir rendu trop de visites à domicile}

Il y a quelque temps déjà, la généraliste de 40 ans avait reçu un courrier de l'organe de surveillance des médecins et des caisses-maladie du land de Hesse. Ce dernier a pour mission de vérifier si les médecins travaillent selon des critères d'économicité, autrement dit si les traitements qu'ils administrent et les médicaments qu'ils prescrivent sont bien - aux termes de la loi - «indispensables et adéquats». Car, après tout, il s'agit de l'argent de la communauté solidaire des assurés. Et maintenant Silvia Steinebach doit justifier par écrit pourquoi elle fait plus d'accompagnements de fin de vie à domicile que la moyenne des médecins en Hesse, avec le risque, si elle ne convainc pas, de devoir rembourser une partie de ses honoraires. «J'adore ma vie à la campagne, et je me sens très attachée à mes pa- tients", nous confie-t-elle, mais après tout ça, elle n'est plus certaine de vouloir rester à Hainzell. D'autres médecins ont connu le même sort: Nils Wagner-Praus et Marei Schoeller, par exemple, qui travaillaient dans un cabinet de groupe à Gilserberg, une commune regroupant onze villages entre Kassel et Francfort. «Il n'y a pas de transports publics chez nous, pas même une entreprise de taxis», nous explique Nils Wagner-Praus. Par contre, il y a beaucoup de personnes âgées. Les deux médecins consultent ainsi souvent à domicile trop selon l'organe de surveillance. Et eux aussi sont astreints à rembourser une partie de leurs honoraires, soit plus de 50000 euros dans leur cas. «Nous sommes consternés", avoue notre médecin. "Un généraliste à Francfort ne consulte que rarement à domicile, car les infrastructures de sa ville permettent aux patients de venir au cabinet.» Il en va autrement à la campagne. A un étudiant en médecine qui effectuait récemment un stage chez lui, il a déconseillé de suivre son exemple.

\section{Surtout, ne deviens pas médecin de campagne}

C'est là un signal déplorable, à une époque où l'on cherche désespérément des médecins de campagne. Autorités, associations d'assureurs et communes rivalisent de créativité pour attirer des jeunes médecins dans les campagnes. Certaines communes construisent des centres de soins communautaires pour permettre de travailler à temps partiel, ou elles se chargent de trouver un emploi pour le ou la partenaire 
du médecin, ou vont jusqu'à proposer une aide au démarrage. Certains lands prévoient de réserver une partie des places en faculté de médecine aux étudiants qui s'engageraient à travailler plus tard dans une région mal desservie.

Mais tout cela ne servira à rien si les médecins en exercice déconseillent à leurs jeunes collègues de s'installer à la campagne. Pour le groupement des médecins conventionnés (KV) de Hesse, les actions récursoires sont en plus contre-productives. "Même si un petit nombre de cabinets seulement y sont astreints, au final une telle procédure représente une lourde charge psychologique, car les sommes en jeu sont parfois considérables», a pu dire leur porte-parole. C'est l'expérience qu'ont faite Marei Schoeller et Nils Wagner-Praus, qui ont vu leur recours rejeté. Dans leur cas, le KV avait estimé que les médecins siégeant dans la commission d'examen indépendante n'avaient trouvé «aucun motif qui puisse justifier sur le plan médical les importants dépassements constatés». Pour Marei Schoeller, «le critère déterminant ne devrait pas être le nombre moyen de visites à domicile. Soit une visite est nécessaire, soit elle ne l'est pas.» Même son de cloche chez Silvia Steinebach, de Hainzell: puisque les autres médecins de campagne rendent moins souvent visite à domicile et font moins d'accompagnement de mourants, il est logique qu'elle apparaisse comme déviant de la statistique. Selon les indications du KV, le nombre de visites à domicile a diminué d'un tiers environ entre 2009 et 2017. Et côté soins palliatifs à domicile, le nombre de visites a diminué de quelque dix pour cent depuis 2014.

Nos deux médecins de Gilserberg n'ont pas eu d'autre choix que de réduire leurs visites à domicile. Et cela depuis quelque temps déjà, car les avertissements se succédaient. Ils avaient pu se défendre jusque-là, en invoquant les particularités de leur région. Mais après des premières demandes de restitution pour la période 2012 à 2014, ils risquent de voir arriver des demandes pour 2015 et 2016. "Nous ne voyons pas comment réduire encore davantage le nombre de nos visites à domicile sans réduire en même temps la qualité des soins", se désole Marei Schoeller. Comment continuer dans ces conditions? Les deux médecins l'ignorent.

\section{C'est l'arbitraire qui dérange le plus}

Petra et Oliver Ranze ont fait leur choix: ils vont émigrer en Suisse. La décision n'a pas été facile, explique Petra Ranze. Mais après avoir été plusieurs fois astreints à des restitutions, bien qu'ils aient fait encore plus attention que leurs collègues à l'argent des assurés et qu'ils n'aient jamais épuisé entièrement leur budget, ils en ont eu assez. Il est même arrivé une fois que, dans le cadre d'un contrôle aléatoire, ils aient dû rembourser 4000 euros pour avoir prescrit trop souvent un inhibiteur de la pompe à protons qui, de l'avis du $\mathrm{KV}$, ne présentait aucun avantage par rapport à d'autres médicaments moins chers. Une autre fois, on leur a réclamé 1000 euros, car ils avaient prescrit trop de physiothérapie. Même s'il s'agit de sommes relativement petites, estime Petra Ranze, «c'est le caractère arbitraire de la procédure qui dérange». Alors, à 57 ans, bien qu'elle ne soit plus dans une situation où elle aimerait recommencer ailleurs une nouvelle carrière, elle a choisi de partir à Zurich pour assumer, dès ce mois de juillet, un poste salarié dans un centre médical.

Source: FAZ du 3 mai 2018, (c) Tous droits réservés. Frankfurter Allgemeine Zeitung $\mathrm{GmbH}$, Francfort. Mis à disposition par les archives du journal.

\section{Référence}

1 Pour en savoir plus sur les budgets globaux et les actions récursoires, nous vous invitons à consulter l'article suivant: Gassen A. Au cœur du piège budgétaire. Bull Med Suisses. 2018;99(39):1306-8. URL: https://bullmed.ch/fr/article/doi/bms.2018.17119/

\section{Le budget global se traduit par un ration-} nement des prestations médicales

Le couple de médecins évoqué dans l'article de la Frankfurter Allgemeine Zeitung a en fait quitté I'Allemagne pour la Suisse. La FMH a demandé au Dr Oliver Ranze ce qui a changé dans son quotidien.

Quelles différences constatez-vous dans votre travail de médecin entre I'Allemagne et la Suisse?

En Suisse, j'ai plus de temps pour mes patients, et j'ai le sentiment d'être apprécié davantage. En général, le médecin de famille est mieux considéré en Suisse dans le système. Quant au tarif ambulatoire, il reflète les prestations médicales de manière assez juste. En tant que médecin, je jouis d'une grande liberté dans le choix des traitements, dans le cadre fixé par les exigences d'économicité, car je ne porte plus personnellement la responsabilité financière du traitement des patients et n'ai plus à craindre $d^{\prime}$ actions récursoires.

Comment expliqueriez-vous à un médecin suisse la manière dont les budgets globaux en Allemagne se répercutent sur la profession, mais aussi sur les patients?

Le budget global se traduit par un rationnement des prestations médicales, notamment pour les patients souffrant d'affections chroniques et nécessitant des traitements particulièrement lourds. Même la fourniture de médicaments et de produits et dispositifs médicaux est impactée. Du fait de ce rationnement, toujours moins de jeunes collègues sont disposés à se lancer dans une carrière de médecin de famille. Mais surtout les zones rurales souffrent de couverture insuffisante, car les budgets globaux accentuent la tendance vers la centralisation des soins médicaux. 